\title{
ON SOME QUASILINEAR WAVE EQUATIONS WITH DISSIPATIVE TERMS
}

\author{
YOSHIO YAMADA
}

\section{§1. Introduction}

In this paper we consider the initial value problems for the following quasilinear wave equations with dissipative terms

$$
u_{t t}-a\left(\int_{R^{n}}|\operatorname{grad} u(x, t)|^{2} d x\right) \Delta u+\lambda u_{t}=f, x \in R^{n}, t \in[0, \infty),
$$

with initial conditions

$$
\begin{array}{ll}
u(x, 0)=u_{0}(x), & x \in R^{n}, \\
u_{t}(x, 0)=u_{1}(x), & x \in R^{n},
\end{array}
$$

where

$$
u_{t}=\frac{\partial u}{\partial t}, u_{t t}=\frac{\partial^{2} u}{\partial t^{2}},|\operatorname{grad} u|^{2}=\sum_{i=1}^{n}\left|\frac{\partial u}{\partial x_{i}}\right|^{2} \text { and } \Delta u=\sum_{i=1}^{n} \frac{\partial^{2} u}{\partial x_{i}^{2}} .
$$

Here $\lambda$ is a positive constant and $a(r)$ is a $C^{1}[0, \infty)$-function satisfying

$$
a(r) \geqq a_{0}>0 \quad \text { for } r \geqq 0 .
$$

For $n=1$, Dickey [3] has treated (1.1) with $\lambda=0$ as the equation describing the small amplitude vibration of a string in which the dependence of the tension on the deformation cannot be neglected. He has shown the existence and uniqueness of local solutions to (1.1)-(1.3) by using a Galerkin procedure. For general $n$, Menzala [6], [7] has recently extended Dickey's result. He has obtained the local existence and uniqueness of classical solutions to (1.1)-(1.3) (with $\lambda=0$ ) by using the theory of Fourier transform. (See also the papers of Dickey [1], [2], Lions [4] and Pohozaev [10], where the mixed problems in a bounded domain are treated.)

Received August 11, 1979. 
The main interest of the present paper is to examine whether there exists a global solution $u$ of (1.1)-(1.3) under the presence of the dissipative term $\lambda u_{t}(\lambda>0)$. Moreover, if such $u$ does exist, we intend to investigate its asymptotic behavior as $t \rightarrow \infty$. The proof of the local solvability of the problem (1.1)-(1.3) is carried out by an iteration procedure (which is different from Menzala's proof). The key point which enables us to extend a local solution $u$ to the interval $[0, \infty)$ lies in deriving some a priori estimates of $u$. Roughly speaking, if the data $\left(u_{0}, u_{1}, f\right)$ are 'small', then there exists a (unique) global solution $u$ of (1.1)-(1.3). Furthermore, by employing the weighted energy method we can obtain the rate of the decay to zero of $u$ as $t \rightarrow \infty$.

The content of this paper is as follows. In $\S 2$, we give our main results: Theorem I (local existence), Theorem II (global existence), Theorem III (regularity of solution) and Theorem IV (asymptotic behavior). $\S 3$ is devoted to the proofs of Theorems I, II and III. In $\S 4$ we study the asymptotic behavior of global solutions of (1.1)-(1.3). Finally, in $\S 5$ some results on the mixed problem are stated without proofs.

The author wishes to express his hearty thanks to Professor H. Tanabe, K. Maruo and A. Yagi for stimulating conversations. Also, the hospitality of Osaka University during the preparation of the manuscript is gratefully acknowledged.

\section{§2. Assumptions and results}

We first introduce some notation which will be used throughout this paper. In the usual way, let $L^{2}\left(R_{x}^{n}\right)$ be the Hilbert space of (complex valued) square integrable functions on $R_{x}^{n}$. The inner product and norm in $L^{2}\left(R_{x}^{n}\right)$ are defined by

$$
(f, g)_{L^{2}\left(R_{x}^{n}\right)}=\int_{R^{n}} f(x) \overline{g(x)} d x \quad \text { for } f, g \in L^{2}\left(R_{x}^{n}\right),
$$

and

$$
\|f\|_{L^{2}\left(R_{x}^{n}\right)}=(f, f)_{L^{2}\left(R_{x}^{n}\right)}^{1 / 2} \quad \text { for } f \in L^{2}\left(R_{x}^{n}\right),
$$

respectively. If there is no confusion, we sometimes write $(\cdot, \cdot)$ (resp. $\|\cdot\|)$ in stead of $(\cdot, \cdot)_{L^{2}\left(R_{x}^{n}\right)}$ (resp. $\left.\|\cdot\|_{L^{2}\left(R_{x}^{n}\right)}\right)$.

For $f \in L^{2}\left(R_{x}^{n}\right)$, define the Fourier transform $\hat{f} \in L^{2}\left(R_{\xi}^{n}\right)$ by 


$$
\begin{aligned}
\hat{f}(\xi) & =(2 \pi)^{-n / 2} \int_{R^{n}} e^{-i x \cdot \xi} f(x) d x, \quad i=\sqrt{-1}, \quad x \cdot \xi=\sum_{i=1}^{n} x_{i} \xi_{i}, \\
& =\underset{A \rightarrow \infty}{1 \cdot \mathrm{i} \cdot \mathrm{m}}(2 \pi)^{-n / 2} \int_{|x| \leqq A} e^{-i x \cdot \xi} f(x) d x,
\end{aligned}
$$

where $1 \cdot \mathbf{i} \cdot \mathrm{m}$ means 'limit in mean'.

For any non-negative integer $s, H^{s}\left(R_{x}^{n}\right)$ denotes the usual Sobolev space of order $s$ with the norm

$$
\|f\|_{s}=\left\{\sum_{i=0}^{s}\||\xi| \hat{f} \hat{f}(\cdot)\|_{L^{2}\left(R_{\xi}^{n}\right)}^{2}\right\}^{1 / 2},
$$

where $|\xi|=\left(\sum_{i=1}^{n} \xi_{i}^{2}\right)^{1 / 2}$. It is very convenient to introduce the following semi-norms;

$$
|f|_{i}=\||\xi| \hat{i} \hat{f}(\cdot)\|_{L^{2}\left(R_{\xi}^{n}\right)}, \quad 0 \leqq i \leqq s,
$$

for $f \in H^{s}\left(R_{x}^{n}\right)$. In particular, if $f \in L^{2}\left(R_{x}^{n}\right)=H^{0}\left(R_{x}^{n}\right)$, then

$$
|f|_{0}=\|f\|_{0}=\|f\|_{L^{2}\left(R_{x}^{n}\right)} \quad \text { (Parseval's equality). }
$$

Let $I$ be any subinterval of $[0, \infty)$. For any Hilbert space $X$, let $C(I ; X)$ be the space of all functions $u: I \rightarrow X$ such that $u$ is strongly continuous on $I$. By $C^{j}(I ; X)$ we denote the space of all functions $u \in$ $C(I ; X)$ such that $u$ is $j$ times strongly continuously differentiable on $I$.

In what follows, we make the following assumptions on the functions $a, u_{0}, u_{1}$ and $f$ appearing in (1.1)-(1.3).

(A.1) The function $a(r)$ for $r \geqq 0$ belongs to the class $C^{1}[0, \infty)$ and satisfies

$$
a(r) \geqq a_{0}>0 \quad \text { for } r \geqq 0 .
$$

(A.2) $u_{0} \in H^{2}\left(R_{x}^{n}\right) \quad$ and $\quad u_{1} \in H^{1}\left(R_{x}^{n}\right)$.

(A.3) $f \in C\left([0, \infty) ; H^{1}\left(R_{x}^{n}\right)\right)$.

Now we shall give our main results. We begin with the local existence theorem.

Theorem I (local existence). There exists a positive constant $T_{0}$ such that the initial value problem (1.1)-(1.3) has a unique solution $u \in C^{i}\left(\left[0, T_{0}\right]\right.$; $\left.H^{2-i}\left(R_{x}^{n}\right)\right)(i=0,1,2)$ on $\left[0, T_{0}\right]$.

Remark 2.1. In general, the constant $T_{0}$ in Theorem I depends on $\left\|u_{0}\right\|_{2},\left\|u_{1}\right\|_{1}$ and $\int_{0}^{T}\|f(s)\|_{1} d s$, where $T$ is any fixed positive number (see (3.2) and (3.3) in $\S 3)$. 
Before stating the global existence theorem, we shall introduce the following set of the data $\left(u_{0}, u_{1}, f\right)$ :

$$
\begin{aligned}
D(\delta)= & \left\{\left(u_{0}, u_{1}, f\right) \in H^{2}\left(R_{x}^{n}\right) \times H^{1}\left(R_{x}^{n}\right) \times C\left([0, \infty) ; H^{1}\left(R_{x}^{n}\right)\right) ;\right. \\
& \left.\left\|u_{0}\right\|_{2} \leqq \delta,\left\|u_{1}\right\|_{1} \leqq \delta \text { and } \int_{0}^{\infty}\|f(s)\|_{1} d s \leqq \delta\right\}
\end{aligned}
$$

We have

TheOREm II (global existence). There exists a positive number $\delta_{0}$ (which depends on $a, a^{\prime}$ and $\left.\lambda\right)$ with the following property: if $\left(u_{0}, u_{1}, f\right) \in D\left(\delta_{0}\right)$, then the initial value problem (1.1)-(1.3) has a unique solution $u \in C^{i}([0, \infty)$; $\left.H^{2-i}\left(R_{x}^{n}\right)\right)(i=0,1,2)$ on $[0, \infty)$. Furthermore,

$$
\sup _{t \geq 0}\|u(t)\|_{2}<\infty \quad \text { and } \quad \sup _{t \geq 0}\left\|u_{t}(t)\right\|_{1}<\infty .
$$

When the data $\left(u_{0}, u_{1}, f\right)$ are regular with respect to $x$, we have

TheOREM III (regularity of solutions). Let $\delta_{0}$ be the positive number in Theorem II. Assume that the data $\left(u_{0}, u_{1}, f\right)$ belong to $D\left(\delta_{0}\right)$ and satisfy

$$
u_{0} \in H^{k+2}\left(R_{x}^{n}\right), u_{1} \in H^{k+1}\left(R_{x}^{n}\right) \quad \text { and } f \in C\left([0, \infty) ; H^{k+1}\left(R_{x}^{n}\right)\right)
$$

for $k \geqq 1$. Then the initial value problem (1.1)-(1.3) has a unique solution $u \in C^{i}\left([0, \infty) ; H^{k+2-i}\left(R_{x}^{n}\right)\right)(i=0,1,2)$. Furthermore, if $f \in L^{1}\left(0, \infty ; H^{k+1}\left(R_{x}^{n}\right)\right)$, then

$$
\sup _{t \geqq 0}\|u(t)\|_{k+2}<\infty \quad \text { and } \quad \sup _{t \geqq 0}\left\|u_{t}(t)\right\|_{k+1}<\infty .
$$

In particular, if $a \in C^{k}[0, \infty)$ and $f \in C^{i}\left([0, \infty) ; H^{k+1-i}\left(R_{x}^{n}\right)\right)(i=0,1,2$, $\cdots, k)$, then $u \in C^{i}\left([0, \infty) ; H^{k+2-i}\left(R_{x}^{n}\right)\right)(i=0,1,2, \cdots, k+2)$.

Corollary 2.1 (existence of classical solutions). Let $\delta_{0}$ be the positive number in Theorem II. If the data $\left(u_{0}, u_{1}, f\right) \in D\left(\delta_{0}\right)$ satisfy

$$
u_{0} \in H^{s+2}\left(R_{x}^{n}\right), u_{1} \in H^{s+1}\left(R_{x}^{n}\right) \quad \text { and } \quad f \in C\left([0, \infty) ; H^{s+1}\left(R_{x}^{n}\right)\right)
$$

with $s=[n / 2]+1$, then the initial value problem (1.1)-(1.3) has a unique classical solution $u \in C^{2}\left(R_{x}^{n} \times[0, \infty)\right)$.

In particular, if $a \in C^{\infty}[0, \infty),\left(u_{0}, u_{1}, f\right) \in C_{0}^{\infty}\left(R_{x}^{n}\right) \times C_{0}^{\infty}\left(R_{x}^{n}\right) \times C^{\infty}\left(R_{x}^{n} \times[0, \infty)\right)$ and $\operatorname{supp} f(\cdot, t)$ is compact for each $t \in[0, \infty)$, then the solution $u$ is $C^{\infty}$ with respect to $(x, t) \in R_{x}^{n} \times[0, \infty)$.

Remark 2.2. In order to obtain the global existence of solutions in 
the class $C^{i}\left([0, \infty) ; H^{k+2-i}\left(R_{x}^{n}\right)\right)(i=0,1,2)$ for $k \geqq 1$ as well as in the class $C^{i}\left([0, \infty) ; H^{2-i}\left(R_{x}^{n}\right)\right)(i=0,1,2)$, we have only to put the same 'smallness' condition (2.1) (with $\left.\delta \leqq \delta_{0}\right)$ on the data $\left(u_{0}, u_{1}, f\right)$. This will be expected from the form of the equation (1.1), in which the nonlinearity is caused by the function $a\left(\|\operatorname{grad} u(t)\|^{2}\right)$.

However, there are different situations in the usual quasilinear wave equations where the nonlinearity is caused by functions of the form $a\left(u, u_{t}, u_{i}\right)\left(u_{i}=\partial u / \partial x_{i}\right)$. For details, see the paper of Matsumura [5].

Finally we shall investigate the asymptotic behavior of global solutions. For simplicity, we assume that the data $\left(u_{0}, u_{1}\right)$ belong to $C_{0}^{\infty}\left(R_{x}^{n}\right)$ and that $f \equiv 0$; we consider

$$
u_{t t}-a\left(\int_{R^{n}}|\operatorname{grad} u(x, t)|^{2} d x\right) \Delta u+\lambda u_{t}=0, x \in R^{n}, t \geqq 0,
$$

with initial conditions (1.2) and (1.3). Let $\left(u_{0}, u_{1}, 0\right)$ be in $D\left(\delta_{0}\right)\left(\delta_{0}\right.$ is the positive number in Theorem II). As for the asymptotic behavior of a solution $u$ of $(1.1)^{\prime},(1.2)$ and $(1.3)$ (which exists globally on $[0, \infty)$ by Theorem III), we have the following result.

Theorem IV (asymptotic behavior). Let $u$ be a global solution of (1.1)' with initial conditions (1.2) and (1.3). Then

$$
\begin{aligned}
& \|u(t)\|^{2}=O(1) \quad \text { as } t \rightarrow \infty, \\
& |u(t)|_{j+1}^{2}+\left|u_{t}(t)\right|_{j}^{2}=O\left(t^{-j-1}\right) \quad \text { as } t \rightarrow \infty, \\
& \left|u_{t t}(t)\right|_{j}^{2}=O\left(t^{-j-1}\right) \quad \text { as } t \rightarrow \infty,
\end{aligned}
$$

for every $j \geqq 0$.

If $a \in C^{2}[0, \infty)$, then

$$
\left|u_{t t}(t)\right|_{j}^{2}=O\left(t^{-j-2}\right) \quad \text { as } t \rightarrow \infty,
$$

for every $j \geqq 0$.

As a consequence of Theorem IV, we can estimate the rate of the decay to zero of global solutions in the supremum norm. Put

$$
\|u\|_{\infty}=\sup _{x \in R^{n}}|u(x)| \quad \text { for } u \in \mathscr{B}\left(R_{x}^{n}\right)
$$

where $\mathscr{B}\left(R_{x}^{n}\right)$ denotes the space of all bounded continuous functions on $R_{x}^{n}$.

Corollary 2.2. Let $u$ be a global solution of $(1.1)^{\prime}$ with initial con- 
ditions (1.2) and (1.3). Then

$$
\begin{aligned}
& \|u(t)\|_{\infty}=O\left(t^{-n / 4}\right), \\
& \left\|u_{i}(t)\right\|_{\infty},\left\|u_{t}(t)\right\|_{\infty},\left\|u_{t t}(t)\right\|_{\infty}=O\left(t^{-(n+2) / 4}\right), \quad 1 \leqq i \leqq n, \\
& \left\|u_{i t}(t)\right\|_{\infty},\left\|u_{i j}(t)\right\|_{\infty}=O\left(t^{-(n+4) / 4}\right), \quad 1 \leqq i, j \leqq n
\end{aligned}
$$

as $t \rightarrow \infty$, where $u_{i}=\partial u / \partial x_{i}, u_{i t}=\partial^{2} u / \partial x_{i} \partial t$ and $u_{i j}=\partial^{2} u / \partial x_{i} \partial x_{j}$.

Remark 2.3. Let $u$ be a global solution of $(1.1)^{\prime}$ with initial conditions (1.2) and (1.3). If the data $\left(u_{0}, u_{1}\right)$ are $C_{0}^{\infty}\left(R_{x}^{n}\right)$-functions, then it is easily seen from Theorem IV that the support of $u(\cdot, t)$ is contained in the ball

$$
\left\{x \in R^{n} ;|x| \leqq \alpha t+C_{\alpha}\right\}
$$

for some $C_{\alpha}$. Here $\alpha$ is an arbitrary number such that $\alpha>a(0)^{1 / 2}$.

\section{§3. Proofs of existence theorems}

In this section we shall prove Theorems I, II and III. We first prepare the following elementary lemma without proof.

LEMMA 3.1. Let $F, G$ and $H$ be non-negative continuous functions on $[0, T](T>0)$. If

$$
F(t)^{2} \leqq \int_{0}^{t} F(s) G(s) d s+H(t), \quad 0 \leqq t \leqq T,
$$

then

$$
F(t) \leqq \frac{1}{2} \int_{0}^{t} G(s) d s+\max _{0 \leqq s \leqq t} H(s)^{1 / 2}, \quad 0 \leqq t \leqq T
$$

\subsection{Proof of Theorem I}

Let an arbitrary $T(>0)$ be fixed. We denote by $K$ the set of all functions $v \in C^{i}\left(\left[0, T_{0}\right] ; H^{2-i}\left(R_{x}^{n}\right)\right)(i=0,1,2)$ such that

$$
v(0)=u_{0} \quad \text { and } \quad v_{t}(0)=u_{1},
$$

and

$$
\|\operatorname{grad} v(t)\|_{1} \leqq N \quad \text { and } \quad\left\|v_{t}(t)\right\|_{1} \leqq N \quad \text { for } 0 \leqq t \leqq T_{0},
$$

where $N$ is a positive constant satisfying

$$
N \geqq \frac{2}{\min \left\{1, a_{0}\right\}}\left\{\left\|u_{1}\right\|_{1}^{2}+a\left(\left\|\operatorname{grad} u_{0}\right\|^{2}\right)\left\|\operatorname{grad} u_{0}\right\|^{2}+\frac{1}{2 \lambda} \int_{0}^{T}\|f(s)\|_{1}^{2} d s\right\},
$$


and $T_{0}(\leqq T)$ is a positive constant satisfying

$$
\exp \left(\frac{2 m N^{2} T_{0}}{a_{0}}\right) \leqq 2 \quad\left(m=\max _{0 \leqq r \leqq N 2}\left|a^{\prime}(r)\right|\right),
$$

and

$$
4 m N^{2} T_{0}<1 .
$$

For each $v \in K$, we consider the initial value problem for

$$
u_{t t}-a\left(\|\operatorname{grad} v(t)\|^{2}\right) \Delta u+\lambda u_{t}=f, x \in R^{n}, t \in\left[0, T_{0}\right],
$$

with initial conditions (1.2) and (1.3). By (A.1) and the definition of $K$, the function $t \rightarrow a\left(\|\operatorname{grad} v(t)\|^{2}\right)$ is continuously differentiable on $\left[0, T_{0}\right]$. Therefore, it is easily seen that there exists a unique solution $u \in C^{i}\left(\left[0, T_{0}\right]\right.$; $\left.H^{2-i}\left(R_{x}^{n}\right)\right)(i=0,1,2)$ of (3.4) satisfying (1.2) and (1.3) (see e.g. Mizohata [8]). We define a mapping $S$ by $u=S v$.

We shall show that $S$ maps $K$ into itself. To see this, we put

$$
u_{\varepsilon}(x, t)=\left(\rho_{\varepsilon} * u\right)(x, t) \equiv \int_{R^{n}} \rho_{\varepsilon}(x-y) u(y, t) d y,
$$

where $\rho_{s} *$ is Friedrichs' mollifier. (For the mollifier, see e.g. Mizohata [8].) Note that $u_{\varepsilon} \in C^{i}\left(\left[0, T_{0}\right] ; H^{s}\left(R_{x}^{n}\right)\right)(i=0,1,2)$ for any $s \geqq 0$. The application of $\rho_{e} *$ to (3.4) gives

$$
u_{\varepsilon, t t}-a\left(\|\operatorname{grad} v(t)\|^{2}\right) \Delta u_{\varepsilon}+\lambda u_{\varepsilon, t}=f_{s}, x \in R^{n}, t \in\left[0, T_{0}\right],
$$

where $f_{\mathrm{s}}(x, t)=\left(\rho_{s} * f\right)(x, t)$. Multiplying (3.6) by $(1-\Delta) \overline{u_{s, t}}$ and integrating over $R_{x}^{n}$, we have

$$
\begin{aligned}
& \frac{1}{2} \frac{d}{d t}\left\{\left\|u_{\varepsilon, t}(t)\right\|_{1}^{2}+a\left(\|\operatorname{grad} v(t)\|^{2}\right)\left\|\operatorname{grad} u_{\varepsilon}(t)\right\|_{1}^{2}\right\}+\lambda\left\|u_{s, t}(t)\right\|_{1}^{2} \\
& =\operatorname{Re}\left\{\left(f_{\varepsilon}(t), u_{\varepsilon, t}(t)\right)+\left(\operatorname{grad} f_{s}(t), \operatorname{grad} u_{\varepsilon, t}(t)\right)\right\} \\
& \quad+a^{\prime}\left(\|\operatorname{grad} v(t)\|^{2}\right) \operatorname{Re}\left(\operatorname{grad} v(t), \operatorname{grad} v_{t}(t)\right)\left\|\operatorname{grad} u_{\varepsilon}(t)\right\|_{1}^{2} \\
& \leqq \lambda\left\|u_{s, t}(t)\right\|_{1}^{2}+\frac{1}{4 \lambda}\left\|f_{\varepsilon}(t)\right\|_{1}^{2}+m N^{2}\left\|\operatorname{grad} u_{s}(t)\right\|_{1}^{2}, \quad 0 \leqq t \leqq T_{0},
\end{aligned}
$$

where we have used Parseval's equality. Hence it follows that

$$
\begin{aligned}
& \left\|u_{\varepsilon, t}(t)\right\|_{1}^{2}+a\left(\|\operatorname{grad} v(t)\|^{2}\right)\left\|\operatorname{grad} u_{\varepsilon}(t)\right\|_{1}^{2} \\
& \leqq\left\|u_{\varepsilon, t}(0)\right\|_{1}^{2}+a\left(\left\|\operatorname{grad} u_{0}\right\|^{2}\right)\left\|\operatorname{grad} u_{\varepsilon}(0)\right\|_{1}^{2} \\
& \quad+\frac{1}{2 \lambda} \int_{0}^{t}\left\|f_{s}(s)\right\|_{1}^{2} d s+2 m N^{2} \int_{0}^{t}\left\|\operatorname{grad} u_{s}(s)\right\|_{1}^{2} d s,
\end{aligned}
$$


for $0 \leqq t \leqq T_{0}$. Letting $\varepsilon \downarrow 0$ in (3.7) and using (A.1) we easily obtain

$$
\begin{aligned}
& \left\|u_{t}(t)\right\|_{1}^{2}+a_{0}\|\operatorname{grad} u(t)\|_{1}^{2} \leqq\left\|u_{1}\right\|_{1}^{2}+a\left(\left\|\operatorname{grad} u_{0}\right\|^{2}\right)\left\|\operatorname{grad} u_{0}\right\|_{1}^{2} \\
& +\frac{1}{2 \lambda} \int_{0}^{t}\|f(s)\|_{1}^{2} d s+2 m N^{2} \int_{0}^{t}\|\operatorname{grad} u(s)\|_{1}^{2} d s, \quad 0 \leqq t \leqq T_{0} ;
\end{aligned}
$$

so that (3.8) implies, by virtue of Gronwall's inequality, that

$$
\begin{aligned}
& \left\|u_{t}(t)\right\|_{1}^{2}+a_{0}\|\operatorname{grad} u(t)\|_{1}^{2} \\
& \leqq\left\{\left\|u_{1}\right\|_{1}^{2}+a\left(\left\|\operatorname{grad} u_{0}\right\|^{2}\right)\left\|\operatorname{grad} u_{0}\right\|_{1}^{2}+\frac{1}{2 \lambda} \int_{0}^{T}\|f(s)\|_{1}^{2} d s\right\} \exp \left(\frac{2 m N^{2} t}{a_{0}}\right), \\
& \quad \text { for } 0 \leqq t \leqq T_{0} .
\end{aligned}
$$

Hence, noting (3.1) and (3.2) we see $u \in K: S$ maps $K$ into itself.

Now we shall construct a local solution of the initial value problem (1.1)-(1.3). Let $u^{0}$ be any element in $K$. Define $\left\{u^{\mu}\right\}_{\mu=0}^{\infty}$ by

$$
u^{\mu+1}=S u^{\mu}, \quad \mu=0,1,2, \cdots .
$$

In other words, $u^{\mu}$ is defined by

$$
u_{t t}^{\mu}-a\left(\left\|\operatorname{grad} u^{\mu-1}(t)\right\|^{2}\right) \Delta u^{\mu}+\lambda u_{t}^{\mu}=f, x \in R^{n}, t \in\left[0, T_{0}\right],
$$

with initial conditions

$$
u^{\mu}(x, 0)=u_{0}(x) \text { and } u_{t}^{\mu}(x, 0)=u_{1}(x), \quad x \in R^{n} .
$$

Since we already know that $S$ maps $K$ into itself,

$$
\left\|\operatorname{grad} u^{\mu}(t)\right\|_{1} \leqq N \text { and }\left\|u_{t}^{\mu}(t)\right\|_{1} \leqq N
$$

for all $\mu \geqq 0$ and $0 \leqq t \leqq T_{0}$. If $w^{\mu}$ is defined by $w^{\mu}=u^{\mu}-u^{\mu-1}$, it is easy to verify that $w^{\mu}$ satisfies

$$
\begin{aligned}
& w_{t t}^{\mu}-a\left(\left\|\operatorname{grad} u^{\mu-1}(t)\right\|^{2}\right) \Delta w^{\mu}+\lambda w_{t}^{\mu} \\
& \quad=\left\{a\left(\left\|\operatorname{grad} u^{\mu-1}(t)\right\|^{2}\right)-a\left(\left\|\operatorname{grad} u^{\mu-2}(t)\right\|^{2}\right)\right\} \Delta u^{\mu-1},
\end{aligned}
$$

( $\left.x \in R^{n}, t \in\left[0, T_{0}\right]\right)$ with initial conditions

$$
w^{\mu}(x, 0)=0 \text { and } w_{t}^{\mu}(x, 0)=0, \quad x \in R^{n} .
$$

Multiplying (3.11) by $\overline{w_{t}^{\mu}}$ and integrating over $R_{x}^{n}$, we get

$$
\begin{aligned}
& \frac{1}{2} \frac{d}{d t}\left\{\left\|w_{t}^{\mu}(t)\right\|^{2}+a\left(\left\|\operatorname{grad} u^{\mu-1}(t)\right\|^{2}\right)\left\|\operatorname{grad} w^{\mu}(t)\right\|^{2}\right\}+\lambda\left\|w_{t}^{\mu}(t)\right\|^{2} \\
& =a^{\prime}\left(\left\|\operatorname{grad} u^{\mu-1}(t)\right\|^{2}\right) \operatorname{Re}\left(\operatorname{grad} u^{\mu-1}(t), \operatorname{grad} u_{t}^{\mu-1}(t)\right)\left\|\operatorname{grad} w^{\mu}(t)\right\|^{2} \\
& \quad+\left\{a\left(\left\|\operatorname{grad} u^{\mu-1}(t)\right\|^{2}\right)-a\left(\left\|\operatorname{grad} u^{\mu-2}(t)\right\|^{2}\right)\right\}\left(\Delta u^{\mu-1}(t), w_{t}^{\mu}(t)\right)
\end{aligned}
$$


for $0 \leqq t \leqq T_{0}$. Since, by (3.10) and Schwarz's inequality,

$$
\left|a^{\prime}\left(\left\|\operatorname{grad} u^{\mu-1}(t)\right\|^{2}\right) \operatorname{Re}\left(\operatorname{grad} u^{\mu-1}(t), \operatorname{grad} u_{t}^{\mu-1}(t)\right)\right| \leqq m N
$$

and

$$
\begin{aligned}
& \left|\left\{a\left(\left\|\operatorname{grad} u^{\mu-1}(t)\right\|^{2}\right)-a\left(\left\|\operatorname{grad} u^{\mu-2}(t)\right\|^{2}\right)\right\}\left(\Delta u^{\mu-1}(t), w_{t}^{\mu}(t)\right)\right| \\
& \quad \leqq 2 m N^{2}\left\|\operatorname{grad} w^{\mu-1}(t)\right\|\left\|w_{t}^{\mu}(t)\right\|,
\end{aligned}
$$

(3.12) leads us to the following inequality:

$$
\begin{aligned}
& \left\|w_{t}^{\mu}(t)\right\|^{2}+a_{0}\left\|\operatorname{grad} w^{\mu}(t)\right\|^{2}+2 \lambda \int_{0}^{t}\left\|w_{t}^{\mu}(s)\right\|^{2} d s \\
& \leqq 2 m N^{2}\left\{\int_{0}^{t}\left\|\operatorname{grad} w^{\mu}(s)\right\|^{2} d s+2 \int_{0}^{t}\left\|\operatorname{grad} w^{\mu-1}(s)\right\|\left\|w_{t}^{\mu}(s)\right\| d s\right\} \\
& \leqq \frac{2 m N^{2}}{a_{0}} \int_{0}^{t}\left(\left\|w_{t}^{\mu}(s)\right\|^{2}+a_{0}\left\|\operatorname{grad} w^{\mu}(s)\right\|^{2}\right) d s \\
& +2 m N^{2} a_{0} \int_{0}^{t}\left\|\operatorname{grad} w^{\mu-1}(s)\right\|^{2} d s, \quad 0 \leqq t \leqq T_{0} .
\end{aligned}
$$

Therefore, applying Gronwall's inequality to (3.13) we have

$$
\begin{gathered}
\left\|w_{t}^{\mu}(t)\right\|^{2}+a_{0}\left\|\operatorname{grad} w^{\mu}(t)\right\|^{2} \leqq 2 m N^{2} T_{0} \cdot \max _{0 \leqq s \leqq T_{0}}\left\{a_{0}\left\|\operatorname{grad} w^{\mu-1}(s)\right\|^{2}\right\} \cdot \exp \left(\frac{2 m N^{2} t}{a_{0}}\right), \\
0 \leqq t \leqq T_{0},
\end{gathered}
$$

from which we deduce that $\left\{u^{\mu}\right\}$ is a Cauchy sequence in $C^{i}\left(\left[0, T_{0}\right] ; H^{1-i}\left(R_{x}^{n}\right)\right)$, $i=0,1$ (see (3.2) and (3.3)). Let $u$ denote the limit of $u^{\mu}$ in $C^{i}\left(\left[0, T_{0}\right]\right.$; $\left.H^{1-i}\left(R_{x}^{n}\right)\right)(i=0,1)$. We can also see from (3.10) that $u^{\mu}(t) \rightarrow u(t)$ (weak convergence) in $H^{2}\left(R_{x}^{n}\right)$ uniformly in $t \in\left[0, T_{0}\right]$ and $u_{t}^{\mu}(t) \rightarrow u_{t}(t)$ in $H^{1}\left(R_{x}^{n}\right)$ uniformly in $t \in\left[0, T_{0}\right]$; so that, in view of $(3.9), u_{t t}^{\mu}(t) \rightarrow u_{t t}(t)$ in $L^{2}\left(R_{x}^{n}\right)$ uniformly in $t \in\left[0, T_{0}\right]$. Thus letting $\mu \rightarrow \infty$ in (3.9) we find that $u$ satisfies

$$
\left(u_{t t}(t), \phi\right)-a\left(\|\operatorname{grad} u(t)\|^{2}\right)(\Delta u(t), \phi)+\lambda\left(u_{t}(t), \phi\right)=(f(t), \phi)
$$

for every $\phi \in L^{2}\left(R_{x}^{n}\right)$ and $t \in\left[0, T_{0}\right]$. Note that the mappings $t \rightarrow u(t), t \rightarrow u_{t}(t)$ and $t \rightarrow u_{t t}(t)$ are weakly continuous in $H^{2}\left(R_{x}^{n}\right), H^{1}\left(R_{x}^{n}\right)$ and $L^{2}\left(R_{x}^{n}\right)$, respectively.

In order to prove $u \in C^{i}\left(\left[0, T_{0}\right] ; H^{2-i}\left(R_{x}^{n}\right)\right)(i=0,1,2)$, we consider the initial value problem for

$$
u_{t t}^{*}-a\left(\|\operatorname{grad} u(t)\|^{2}\right) \Delta u^{*}+\lambda u_{t}^{*}=f, x \in R^{n}, t \in\left[0, T_{0}\right],
$$

with initial conditions (1.2) and (1.3). Since the function $t \rightarrow a\left(\|\operatorname{grad} u(t)\|^{2}\right)$ 
is continuously differentiable on $\left[0, T_{0}\right]$, the initial value problem (3.15), (1.2) and (1.3) has a unique solution $u^{*} \in C^{i}\left(\left[0, T_{0}\right] ; H^{2-i}\left(R_{x}^{n}\right)\right), i=0,1,2$. Put $w=u-u^{*}$; then, by (3.14) and (3.15), $w$ satisfies the equation

$$
\left(w_{t t}(t), \phi\right)-a\left(\|\operatorname{grad} u(t)\|^{2}\right)(\Delta w(t), \phi)+\lambda\left(w_{t}(t), \phi\right)=0,
$$

for every $\phi \in L^{2}\left(R_{x}^{n}\right)$ and $t \in\left[0, T_{0}\right]$ with zero initial data. Hence, setting $\phi=w_{t}$ in (3.16) and integrating over $[0, t]\left(0 \leqq t \leqq T_{0}\right)$, we have

$$
\begin{aligned}
& \left\|w_{t}(t)\right\|^{2}+a\left(\|\operatorname{grad} u(t)\|^{2}\right)\|\operatorname{grad} w(t)\|^{2}+2 \lambda \int_{0}^{t}\left\|w_{t}(s)\right\|^{2} d s \\
& =2 \int_{0}^{t} a^{\prime}\left(\|\operatorname{grad} u(s)\|^{2}\right) \operatorname{Re}\left(\operatorname{grad} u(s), \operatorname{grad} u_{t}(s)\right)\|\operatorname{grad} w(s)\|^{2} d s,
\end{aligned}
$$

which assures $w \equiv 0$ (i.e. $u=u^{*}$ ) with the aid of Gronwall's inequality. Thus we have shown the existence of a function $u \in C^{i}\left(\left[0, T_{0}\right] ; H^{2-i}\left(R_{x}^{n}\right)\right)$ $(i=0,1,2)$ satisfying (1.1)-(1.3).

Finally we shall prove the uniqueness of local solutions. Let $u, v \in$ $C^{i}\left(\left[0, T_{0}\right] ; H^{2-i}\left(R_{x}^{n}\right)\right)(i=0,1,2)$ be two solutions of the initial value problem (1.1)-(1.3). Put $w^{*}=u-v$; then

$$
\begin{gathered}
\frac{1}{2} \frac{d}{d t}\left\{\left\|w_{t}^{*}(t)\right\|^{2}+a\left(\|\operatorname{grad} u(t)\|^{2}\right)\left\|\operatorname{grad} w^{*}(t)\right\|^{2}\right\}+\lambda\left\|w_{t}^{*}(t)\right\|^{2} \\
=a^{\prime}\left(\|\operatorname{grad} u(t)\|^{2}\right) \operatorname{Re}\left(\operatorname{grad} u(t), \operatorname{grad} u_{t}(t)\right)\left\|\operatorname{grad} w^{*}(t)\right\|^{2} \\
+\left\{a\left(\|\operatorname{grad} u(t)\|^{2}\right)-a\left(\|\operatorname{grad} v(t)\|^{2}\right)\right\}\left(\Delta v(t), w_{t}^{*}(t)\right),
\end{gathered}
$$

(cf. (3.12)). Since $u, v \in C^{i}\left(\left[0, T_{0}\right] ; H^{2-i}\left(R_{x}^{n}\right)\right)(i=0,1,2)$, by integrating (3.17) over $[0, t]$ and applying Gronwall's inequality we may conclude $w^{*} \equiv 0$ on $\left[0, T_{0}\right]$, which completes the proof.

Remark 3.1. Our method of the proof of the local existence theorem is different from that of Menzala [7]. His proof is based on the use of Fourier transforms; the original problem (1.1)-(1.3) is equivalent to the following problem

$$
\begin{cases}\hat{u}_{t t}(\xi, t)+a\left(\||\xi| \hat{u}(\cdot, t)\|^{2}\right)|\xi|^{2} \hat{u}(\xi, t)+\lambda \hat{u}_{t}(\xi, t)=\hat{f}(\xi, t), & \xi \in R^{n}, t \geqq 0, \\ \hat{u}(\xi, 0)=\hat{u}_{0}(\xi), & \xi \in R^{n}, \\ \hat{u}_{t}(\xi, 0)=\hat{u}_{1}(\xi), & \xi \in R^{n},\end{cases}
$$

where $\hat{u}$ denotes the Fourier transform of $u$ (with respect to $x$ ). To approach (3.18), Menzala defined approximate functions $\left\{v_{r}(\xi, t)\right\}\left(v_{r}(\xi, t) \equiv\right.$ 0 for $|\xi| \geqq r)$ as a solution of the truncated problem 


$$
\begin{cases}v_{r, t t}(\xi, t)+a\left(\int_{|\xi| \leqq r}|\xi|^{2}\left|v_{r}(\xi, t)\right|^{2} d \xi\right)|\xi|^{2} v_{r}(\xi, t)+\lambda v_{r, t}(\xi, t)=\hat{f}(\xi, t), \\ v_{r}(\xi, 0)=\hat{u}_{0}(\xi), & |\xi| \leqq r, \quad t \geqq 0, \\ v_{r, t}(\xi, 0)=\hat{u}_{1}(\xi), & |\xi| \leqq r,\end{cases}
$$

Then letting $r \rightarrow \infty$, he has constructed a local solution of (3.18).

See also the paper of Dickey [3].

3.2. Proof of Theorem II

Since the local existence result (Theorem I) is obtained, it suffices to get a priori bounds for any solution of (1.1)-(1.3) in order to show the global existence.

Let $T$ be any fixed positive number and let $u \in C^{i}\left([0, T] ; H^{2-i}\left(R_{x}^{n}\right)\right)$ $(i=0,1,2)$ be a solution of (1.1)-(1.3) on $[0, T]$. Assume that the data $\left(u_{0}, u_{1}, f\right)$ belong to $D(\delta)$ (see (2.1)). We shall show that, if $\delta>0$ is sufficiently small, then both $\|u(t)\|_{2}$ and $\left\|u_{t}(t)\right\|_{1}$ are bounded by a positive number which is independent of $T$, so that $u$ may be extended to the interval $[0, \infty)$.

First multiplying (1.1) by $\bar{u}_{t}$ and integrating over $R_{x}^{n}$, we get

$$
\begin{aligned}
\frac{1}{2} \frac{d}{d t}\left\{\left\|u_{t}(t)\right\|^{2}+A\left(\|\operatorname{grad} u(t)\|^{2}\right)\right\}+\lambda\left\|u_{t}(t)\right\|^{2} & =\operatorname{Re}\left(f(t), u_{t}(t)\right) \\
& \leqq\|f(t)\|\left\|u_{t}(t)\right\|,
\end{aligned}
$$

where $A(r)=\int_{0}^{r} a(s) d s$ ( $\geqq a_{0} r$ for $\left.r \geqq 0\right)$. Integration of (3.19) with respect to $t$ leads to

$$
\begin{aligned}
& \left\|u_{t}(t)\right\|^{2}+a_{0}\|\operatorname{grad} u(t)\|^{2}+2 \lambda \int_{0}^{t}\left\|u_{t}(s)\right\|^{2} d s \\
& \quad \leqq\left\|u_{1}\right\|^{2}+A\left(\left\|\operatorname{grad} u_{0}\right\|^{2}\right)+2 \int_{0}^{t}\|f(s)\|\left\|u_{t}(s)\right\| d s, \quad 0 \leqq t \leqq T .
\end{aligned}
$$

Consequently, applying Lemma 3.1 to (3.20) we have

$$
\left\|u_{t}(t)\right\|^{2}+a_{0}\|\operatorname{grad} u(t)\|^{2}+2 \lambda \int_{0}^{t}\left\|u_{t}(s)\right\|^{2} d s \leqq C_{1}(\delta)^{2},
$$

where $C_{1}(\delta)=\left\{\delta^{2}+A\left(\delta^{2}\right)\right\}^{1 / 2}+\delta$. Next we multiply (1.1) by $\bar{u}$ and integrate over $R_{x}^{n}$. Then

$$
\begin{aligned}
& \operatorname{Re} \frac{d}{d t}\left(u_{t}(t), u(t)\right)+a\left(\|\operatorname{grad} u(t)\|^{2}\right)\|\operatorname{grad} u(t)\|^{2}+\frac{\lambda}{2} \frac{d}{d t}\|u(t)\|^{2} \\
& \quad=\left\|u_{t}(t)\right\|^{2}+\operatorname{Re}(f(t), u(t)), \quad 0 \leqq t \leqq T,
\end{aligned}
$$


from which it follows that

$$
\begin{aligned}
\lambda\|u(t)\|^{2} & +2 a_{0} \int_{0}^{t}\|\operatorname{grad} u(s)\|^{2} d s \leqq \lambda\left\|u_{0}\right\|^{2}+2\left\|u_{1}\right\|\left\|u_{0}\right\|+2\left\|u_{t}(t)\right\|\|u(t)\| \\
& +2 \int_{0}^{t}\left\|u_{t}(s)\right\|^{2} d s+2 \int_{0}^{t}\|f(s)\|\|u(s)\| d s \\
\leqq & (\lambda+2) \delta^{2}+\frac{\lambda}{2}\|u(t)\|^{2}+\frac{2}{\lambda}\left\|u_{t}(t)\right\|^{2}+2 \int_{0}^{t}\left\|u_{t}(s)\right\|^{2} d s \\
& +2 \int_{0}^{t}\|f(s)\|\|u(s)\| d s .
\end{aligned}
$$

Therefore, combining (3.21) and (3.22) we obtain

$$
\begin{aligned}
\frac{\lambda}{2}\|u(t)\|^{2}+2 a_{0} \int_{0}^{t}\|\operatorname{grad} u(s)\|^{2} d s \leqq(\lambda+2) \delta^{2}+\frac{2}{\lambda} C_{1}(\delta)^{2} \\
+2 \int_{0}^{t}\|f(s)\|\|u(s)\| d s, \quad 0 \leqq t \leqq T
\end{aligned}
$$

which implies, with the use of Lemma 3.1, the existence of a positive constant $C_{0}(\delta)$ (independent of $T$ ) such that

$$
\lambda\|u(t)\|^{2}+4 a_{0} \int_{0}^{t}\|\operatorname{grad} u(s)\|^{2} d s \leqq C_{0}(\delta)^{2}
$$

for $0 \leqq t \leqq T$. (Note that the estimates (3.21) and (3.23) hold for any $\left(u_{0}, u_{1}, f\right) \in H^{1}\left(R_{x}^{n}\right) \times L^{2}\left(R_{x}^{n}\right) \times L^{1}\left(0, \infty ; L^{2}\left(R_{x}^{n}\right)\right)$.)

Now in order to estimate $\|u(t)\|_{2}$ and $\left\|u_{t}(t)\right\|_{1}$, it is convenient to employ Friedrichs' mollifier. If $u_{\varepsilon}$ is defined by (3.5), then it satisfies

$$
u_{\varepsilon, t t}-a\left(\|\operatorname{grad} u(t)\|^{2}\right) \Delta u_{\varepsilon}+\lambda u_{\varepsilon, t}=f_{\varepsilon}, \quad x \in R^{n}, \quad t \in[0, T],
$$

where $f_{\varepsilon}=\left(\rho_{\varepsilon} * f\right)$. Multiplying (3.24) by $-\Delta \overline{u_{\varepsilon, t}}$ and integrating over $R_{x}^{n}$, we get

$$
\begin{aligned}
\frac{1}{2} \frac{d}{d t}\left\{\left\|\operatorname{grad} u_{\varepsilon, t}(t)\right\|^{2}+a\left(\|\operatorname{grad} u(t)\|^{2}\right)\left\|\Delta u_{\varepsilon}(t)\right\|^{2}\right\}+\lambda\left\|\operatorname{grad} u_{\varepsilon, t}(t)\right\|^{2} \\
=a^{\prime}\left(\|\operatorname{grad} u(t)\|^{2}\right) \operatorname{Re}\left(\operatorname{grad} u(t), \operatorname{grad} u_{t}(t)\right)\left\|\Delta u_{\varepsilon}(t)\right\|^{2} \\
\quad+\operatorname{Re}\left(\operatorname{grad} f_{\epsilon}(t), \operatorname{grad} u_{\varepsilon, t}(t)\right)
\end{aligned}
$$

Integration of (3.25) with respect to $t$ gives

$$
\begin{aligned}
& \left\|\operatorname{grad} u_{\varepsilon, t}(t)\right\|^{2}+a\left(\|\operatorname{grad} u(t)\|^{2}\right)\left\|\Delta u_{s}(t)\right\|^{2}+2 \lambda \int_{0}^{t}\left\|\operatorname{grad} u_{\varepsilon, t}(s)\right\|^{2} d s \\
& \quad=\left\|\operatorname{grad} u_{\varepsilon, t}(0)\right\|^{2}+a\left(\left\|\operatorname{grad} u_{0}\right\|^{2}\right)\left\|\Delta u_{\varepsilon}(0)\right\|^{2}
\end{aligned}
$$




$$
\begin{aligned}
& +2 \operatorname{Re} \int_{0}^{t}\left(\operatorname{grad} f_{s}(s), \operatorname{grad} u_{s, t}(s)\right) d s \\
& +2 \operatorname{Re} \int_{0}^{t} a^{\prime}\left(\|\operatorname{grad} u(s)\|^{2}\right)\left(\operatorname{grad} u(s), \operatorname{grad} u_{t}(s)\right)\left\|\Delta u_{\mathrm{s}}(s)\right\|^{2} d s
\end{aligned}
$$

Hence by letting $\varepsilon \downarrow 0$ it easily follows that

$$
\begin{gathered}
\left\|\operatorname{grad} u_{t}(t)\right\|^{2}+a\left(\|\operatorname{grad} u(t)\|^{2}\right)\|\Delta u(t)\|^{2}+2 \lambda \int_{0}^{t}\left\|\operatorname{grad} u_{t}(s)\right\|^{2} d s \\
=\left\|\operatorname{grad} u_{1}\right\|^{2}+a\left(\left\|\operatorname{grad} u_{0}\right\|^{2}\right)\left\|\Delta u_{0}\right\|^{2}+2 \operatorname{Re} \int_{0}^{t}\left(\operatorname{grad} f(s), \operatorname{grad} u_{t}(s)\right) d s \\
\quad+2 \operatorname{Re} \int_{0}^{t} a^{\prime}\left(\|\operatorname{grad} u(s)\|^{2}\right)\left(\operatorname{grad} u(s), \operatorname{grad} u_{t}(s)\right)\|\Delta u(s)\|^{2} d s
\end{gathered}
$$

holds for $0 \leqq t \leqq T$. Using (A. 1) and (3.21) we rearrange (3.26); then

$$
\begin{aligned}
& \left\|\operatorname{grad} u_{t}(t)\right\|^{2}+a_{0}\|\Delta u(t)\|^{2}+2 \lambda \int_{0}^{t}\left\|\operatorname{grad} u_{t}(s)\right\|^{2} d s \\
& \leqq \\
& \delta^{2}\left(1+m_{0}\left(\delta^{2}\right)\right)+2 \int_{0}^{t}\|\operatorname{grad} f(s)\|\left\|\operatorname{grad} u_{t}(s)\right\| d s \\
& \quad+\frac{2 m_{1}\left(C_{1}(\delta)^{2} / a_{0}\right) C_{1}(\delta)}{\sqrt{a_{0}}} \int_{0}^{t}\left\|\operatorname{grad} u_{t}(s)\right\|\|\Delta u(s)\|^{2} d s,
\end{aligned}
$$

$(0 \leqq t \leqq T)$, where $m_{0}(r)=\max _{0 \leqq s \leqq r} a(s)$ and $m_{1}(r)=\max _{0 \leqq s \leqq r}\left|a^{\prime}(s)\right|$.

If we multiply (3.24) by $-\overline{\Delta u_{\varepsilon}}$ and integrate over $R_{x}^{n}$, we have

$$
\begin{aligned}
\frac{d}{d t}\{ & \left.\operatorname{Re}\left(\operatorname{grad} u_{\varepsilon, t}(t), \operatorname{grad} u_{\varepsilon}(t)\right)+\frac{\lambda}{2}\left\|\operatorname{grad} u_{\varepsilon}(t)\right\|^{2}\right\} \\
& \quad+a\left(\|\operatorname{grad} u(t)\|^{2}\right)\left\|\Delta u_{\varepsilon}(t)\right\|^{2} \\
= & \left\|\operatorname{grad} u_{\varepsilon, t}(t)\right\|^{2}+\operatorname{Re}\left(\operatorname{grad} f_{s}(t), \operatorname{grad} u_{\varepsilon}(t)\right), \quad 0 \leqq t \leqq T .
\end{aligned}
$$

Integrating (3.28) over $[0, t]$ and letting $\varepsilon \downarrow 0$ in the resulting expression we get

$$
\begin{gathered}
\operatorname{Re}\left(\operatorname{grad} u_{t}(t), \operatorname{grad} u(t)\right)+\frac{\lambda}{2}\|\operatorname{grad} u(t)\|^{2}+\int_{0}^{t} a\left(\|\operatorname{grad} u(s)\|^{2}\right)\|\Delta u(s)\|^{2} d s \\
=\operatorname{Re}\left(\operatorname{grad} u_{1}, \operatorname{grad} u_{0}\right)+\frac{\lambda}{2}\left\|\operatorname{grad} u_{0}\right\|^{2}+\int_{0}^{t}\left\|\operatorname{grad} u_{t}(s)\right\|^{2} d s \\
\quad+\operatorname{Re} \int_{0}^{t}(\operatorname{grad} f(s), \operatorname{grad} u(s)) d s, \quad 0 \leqq t \leqq T
\end{gathered}
$$

By using (2.1) and (3.21) we rearrange (3.29); then 


$$
\begin{gathered}
-\left\|\operatorname{grad} u_{t}(t)\right\|\|\operatorname{grad} u(t)\|+\frac{\lambda}{2}\|\operatorname{grad} u(t)\|^{2}+a_{0} \int_{0}^{t}\|\Delta u(s)\|^{2} d s \\
\leqq\left(1+\frac{\lambda}{2}\right) \delta^{2}+\frac{\delta C_{1}(\delta)}{\sqrt{a_{0}}}+\int_{0}^{t}\left\|\operatorname{grad} u_{t}(s)\right\|^{2} d s
\end{gathered}
$$

for $0 \leqq t \leqq T$. Addition of (3.27) and (3.30) $\times \lambda$ yields

$$
\begin{aligned}
\frac{1}{2} \| \operatorname{grad} & u_{t}(t)\left\|^{2}+a_{0}\right\| \Delta u(t) \|^{2}+\frac{1}{2}\left(\left\|\operatorname{grad} u_{t}(t)\right\|-\lambda\|\operatorname{grad} u(t)\|\right)^{2} \\
& +\lambda \int_{0}^{t}\left\|\operatorname{grad} u_{t}(s)\right\|^{2} d s \\
& +\int_{0}^{t}\left\{\lambda a_{0}-\frac{2 m_{1}\left(C_{1}(\delta)^{2} / a_{0}\right) C_{1}(\delta)}{\sqrt{a_{0}}}\left\|\operatorname{grad} u_{t}(s)\right\|\right\}\|\Delta u(s)\|^{2} d s \\
\leqq & \delta^{2}\left(1+m_{0}\left(\delta^{2}\right)+\lambda+\frac{\lambda^{2}}{2}\right)+\frac{\lambda \delta C_{1}(\delta)}{\sqrt{a_{0}}} \\
& +2 \int_{0}^{t}\|\operatorname{grad} f(s)\|\left\|\operatorname{grad} u_{t}(s)\right\| d s, \quad 0 \leqq t \leqq T .
\end{aligned}
$$

Now suppose that the inequality

$$
\lambda a_{0}^{3 / 2}-2 m_{1}\left(C_{1}(\delta)^{2} / a_{0}\right) C_{1}(\delta)\left\|\operatorname{grad} u_{t}(t)\right\| \geqq 0
$$

holds on $[0, \tau](0 \leqq \tau \leqq T)$. Then applying Lemma 3.1 to (3.31) we have

$$
\begin{aligned}
& \left\|\operatorname{grad} u_{t}(t)\right\|^{2}+2 a_{0}\|\Delta u(t)\|^{2} \\
& \quad \leqq\left[\left\{\delta^{2}\left(2+2 m_{0}\left(\delta^{2}\right)+2 \lambda+\lambda^{2}\right)+\frac{2 \lambda \delta C_{1}(\delta)}{\sqrt{a_{0}}}\right\}^{1 / 2}+2 \int_{0}^{t}\|\operatorname{grad} f(s)\| \mathrm{ds}\right]^{2} \\
& \quad \leqq C_{2}(\delta)^{2}, \quad \text { for } 0 \leqq t \leqq \tau,
\end{aligned}
$$

where $C_{2}(\delta)=\left\{\delta^{2}\left(2+2 m_{0}\left(\delta^{2}\right)+2 \lambda+\lambda^{2}\right)+2 \lambda \delta C_{1}(\delta) / \sqrt{a_{0}}\right\}^{1 / 2}+2 \delta$. Notice that $C_{i}(\delta)(i=1,2)$ are increasing functions of $\delta$ satisfying $C_{i}(0)=0$. Hence it is possible to choose $\delta_{0}$ as a (unique) solution of

$$
\lambda a_{0}^{3 / 2}=4 m_{1}\left(C_{1}\left(\delta_{0}\right)^{2} / a_{0}\right) C_{1}\left(\delta_{0}\right) C_{2}\left(\delta_{0}\right) .
$$

Consequently, it is easily verified that, for $\left(u_{0}, u_{1}, f\right) \in D(\delta)$ with $\delta \leqq \delta_{0}$, $u$ satisfies (3.32) and, therefore, (3.33) on $[0, T]$. Thus we have obtained a priori bounds (3.21), (3.23) and (3.33). So we can conclude in the standard way that the initial value problem (1.1)-(1.3) has a solution $u \in C^{i}([0, \infty)$; $\left.H^{2-i}\left(R_{x}^{n}\right)\right), i=0,1,2$, satisfying (2.2).

The uniqueness part is evident from Theorem I.

3.3. Proof of Theorem III

First we note the following result whose proof is essentially the same 
as that of Theorem I: for each $u_{0} \in H^{k+2}\left(R_{x}^{n}\right), u_{1} \in H^{k+1}\left(R_{x}^{n}\right)$ and $f \in C([0, \infty)$; $\left.H^{k+1}\left(R_{x}^{n}\right)\right)$ with $k \geqq 1$, there exists a positive constant $T_{0}$ such that the initial value problem (1.1)-(1.3) has a unique solution $u \in C^{i}\left(\left[0, T_{0}\right] ; H^{k+2-i}\left(R_{x}^{n}\right)\right)$ $(i=0,1,2)$ on $\left[0, T_{0}\right]$. Hence, in order to prove the existence of a global solution in the class $C^{i}\left([0, \infty) ; H^{k+2-i}\left(R_{x}^{n}\right)\right)(i=0,1,2)$, it suffices to get a priori bounds for $\|u(t)\|_{k+2}$ and $\left\|u_{t}(t)\right\|_{k+1}$.

Let $T$ be any fixed positive number and let $u \in C^{i}\left([0, T] ; H^{k+2-i}\left(R_{x}^{n}\right)\right)$ $(i=0,1,2)$ be a solution of (1.1)-(1.3) on [0,T]. Take $\delta_{0}$ as the positive number in Theorem II and assume $\left(u_{0}, u_{1}, f\right) \in D\left(\delta_{0}\right)$. (Recall that the estimates (3.21), (3.23) and (3.33) hold true with $\delta=\delta_{0}$.)

As in 3.2 , we multiply (3.24) by $(-\Delta)^{j} \bar{u}_{\varepsilon, t}(2 \leqq j \leqq k+1)$ and integrate over $R_{x}^{n}$. Then, by Parseval's equality,

$$
\begin{gathered}
\frac{1}{2} \frac{d}{d t}\left\{\left|u_{s, t}(t)\right|_{j}^{2}+a\left(\|\operatorname{grad} u(t)\|^{2}\right)\left|u_{\varepsilon}(t)\right|_{j+1}^{2}\right\}+\lambda\left|u_{\varepsilon, t}(t)\right|_{j}^{2} \\
=a^{\prime}\left(\|\operatorname{grad} u(t)\|^{2}\right) \operatorname{Re}\left(\operatorname{grad} u(t), \operatorname{grad} u_{t}(t)\right)\left|u_{\varepsilon}(t)\right|_{j+1}^{2} \\
\quad+\operatorname{Re}\left((-\Delta)^{j / 2} f_{s}(t),(-\Delta)^{j / 2} u_{\varepsilon, t}(t)\right)
\end{gathered}
$$

(cf. (3.25)). Integrating the above equality over $[0, t]$ and letting $\varepsilon \downarrow 0$, we deduce

$$
\begin{aligned}
& \left|u_{t}(t)\right|_{j}^{2}+a_{0}|u(t)|_{j+1}^{2}+2 \lambda \int_{0}^{t}\left|u_{t}(s)\right|_{j}^{2} d s \\
& \leqq\left|u_{1}\right|_{j}^{2}+a\left(\left|u_{0}\right|_{1}^{2}\right)\left|u_{0}\right|_{j+1}^{2}+2 \int_{0}^{t}|f(s)|_{j} \cdot\left|u_{t}(s)\right|_{j} d s \\
& \quad+\frac{2 m_{1}\left(C_{1}\left(\delta_{0}\right)^{2} / a_{0}\right) C_{1}\left(\delta_{0}\right) C_{2}\left(\delta_{0}\right)}{\sqrt{a_{0}}} \int_{0}^{t}|u(s)|_{j+1}^{2} d s
\end{aligned}
$$

where we have used (3.21) and (3.33).

If we multiply $(3.24)$ by $(-\Delta)^{j} \bar{u}_{\varepsilon}(2 \leqq j \leqq k+1)$ and integrate over $R_{x}^{n}$, we have

$$
\begin{aligned}
& \frac{d}{d t}\left\{\operatorname{Re}\left((-\Delta)^{j / 2} u_{\varepsilon, t}(t),(-\Delta)^{j / 2} u_{\varepsilon}(t)\right)+\frac{\lambda}{2}\left|u_{\varepsilon}(t)\right|_{j}^{2}\right\}+a\left(\|\operatorname{grad} u(t)\|^{2}\right)\left|u_{\varepsilon, t}(t)\right|_{j+1}^{2} \\
& \quad=\left|u_{\varepsilon, t}(t)\right|_{j}^{2}+\operatorname{Re}\left((-\Delta)^{j / 2} f_{s}(t),(-\Delta)^{j / 2} u_{s}(t)\right)
\end{aligned}
$$

(cf. (3.28)). Hence, it follows by integrating with respect to $t$ and letting $\varepsilon \downarrow 0$ that

$$
\begin{aligned}
& -\left|u_{t}(t)\right|_{j} \cdot|u(t)|_{j}+\frac{\lambda}{2}|u(t)|_{j}^{2}+a_{0} \int_{0}^{t}|u(s)|_{j+1}^{2} d s \\
& \leqq\left|u_{1}\right|_{j} \cdot\left|u_{0}\right|_{j}+\frac{\lambda}{2}\left|u_{0}\right|_{j}^{2}+\int_{0}^{t}\left|u_{t}(s)\right|_{j}^{2} d s+\int_{0}^{t}|f(s)|_{j} \cdot|u(s)|_{j} d s
\end{aligned}
$$


for $0 \leqq t \leqq T$.

Addition of (3.35) and (3.36) $\times \lambda$ yields

$$
\begin{gathered}
\frac{1}{2}\left|u_{t}(t)\right|_{j}^{2}+a_{0}|u(t)|_{j+1}^{2} \leqq\left|u_{1}\right|_{j}^{2}+a\left(\left|u_{0}\right|_{1}^{2}\right)\left|u_{0}\right|_{j+1}^{2}+\lambda\left|u_{1}\right|_{j} \cdot\left|u_{0}\right|_{j}+\frac{\lambda^{2}}{2}\left|u_{0}\right|_{j}^{2} \\
+\int_{0}^{t}|f(s)|_{j}\left(2\left|u_{t}(s)\right|_{j}+\lambda|u(s)|_{j}\right) d s, \quad 0 \leqq t \leqq T,
\end{gathered}
$$

(cf. (3.31)), where we have used (3.34). Therefore, with the aid of Lemma 3.1, (3.37) gives inductively

$$
\left|u_{t}(t)\right|_{j}^{2}+2 a_{0}|u(t)|_{j+1}^{2} \leqq C_{j+1}, \quad 0 \leqq t \leqq T,
$$

for some $C_{j+1}$. In particular, if $f \in L^{1}\left(0, \infty ; H^{k+1}\left(R_{x}^{n}\right)\right)$, then $C_{j+1}$ can be taken independent of $T$; so that (2.3) holds. Thus the first half of Theorem III is proved.

Finally we shall show the latter half. Assume $a \in C^{k}[0, \infty)$ and $f \in C^{i}\left([0, \infty) ; H^{k+1-i}\left(R_{x}^{n}\right)\right)(i=0,1,2, \cdots, k)$. Then, differentiating both sides of (1.1) with respect to $t$, we may conclude that $u$ belongs to the class $C^{i}\left([0, \infty) ; H^{k+2-i}\left(R_{x}^{n}\right)\right)(i=0,1,2, \cdots, k+2)$.

\subsection{Proof of Corollary 2.1.}

By Sobolev's lemma, $H^{m}\left(R_{x}^{n}\right)$ is imbedded in $\mathscr{B}\left(R_{x}^{n}\right)$ if $m \geqq[n / 2]+1$ (see e.g. Sobolev [11] or Mizohata [8]). Therefore, all the conclusions of this corollary are evident from Theorem III.

\section{§4. Asymptotic behavior}

In this section we shall consider the asymptotic behavior of solutions to the equations

$$
u_{t t}-a\left(\|\operatorname{grad} u(t)\|^{2}\right) \Delta u+\lambda u_{t}=0, \quad x \in R^{n}, \quad t \in[0, \infty),
$$

with initial conditions (1.2) and (1.3). For simplicity, we assume that the data $\left(u_{0}, u_{1}\right)$ are $C_{0}^{\infty}\left(R_{x}^{n}\right)$-functions. Moreover, assume that they satisfy

$$
\left\|u_{0}\right\|_{2} \leqq \delta_{0} \text { and }\left\|u_{1}\right\|_{1} \leqq \delta_{0},
$$

where $\delta_{0}$ is the positive constant in Theorem II.

We already know by Theorems II and III that there exists a unique solution $u \in C^{i}\left([0, \infty) ; H^{k+2-i}\left(R_{x}^{n}\right)\right)(i=0,1,2)$ satisfying

$$
\sup _{t \geqq 0}\|u(t)\|_{k+2}<\infty \text { and } \sup _{t \geqq 0}\left\|u_{t}(t)\right\|_{k+1}<\infty \text {, }
$$


for any $k \geqq 0$.

However, we shall show that, because of the presence of the dissipative term $\lambda u_{t}$, both $|u(t)|_{j+1}$ and $\left|u_{t}(t)\right|_{j}$ decay to zero as $t \rightarrow \infty$ for every $j \geqq 0$ (Theorem IV). $\quad(\|u(t)\|$ may not decay to zero as $t \rightarrow \infty$.) Hence, by making use of Nirenberg's inequality (see (4.12)), we can prove that the solution $u$ itself also decays to zero in the supremum norm as $t \rightarrow \infty$ (Corollary 2.2).

\subsection{Proof of Theorem IV}

By (3.23), it is easy to see (2.4). Moreover, by (3.21) and (3.23), there exists a positive constant $C$ such that

$$
\|u(t)\|_{2} \leqq C \text { and }\left\|u_{t}(t)\right\|_{1} \leqq C
$$

for all $t \geqq 0$.

Now we shall prove the following stronger result than (2.5):

$$
\begin{gathered}
t^{j+1}\left(\left|u_{t}(t)\right|_{j}^{2}+|u(t)|_{j+1}^{2}\right)+\int_{0}^{t} s^{j+1}\left|u_{t}(s)\right|_{j}^{2} d s+\int_{0}^{t} s^{j}|u(s)|_{j+1}^{2} d s \leqq M_{j}^{2}, \\
t \geqq 0, j=0,1,2, \cdots,
\end{gathered}
$$

with some $M_{j}>0$.

In order to show (4.2) we employ the weighted energy method. Multiplying (3.19) (with $f \equiv 0$ ) by $t$ and integrating over $[0, t]$ we have

$$
\begin{array}{r}
t\left|u_{t}(t)\right|^{2}+t A\left(|u(t)|_{1}^{2}\right)+2 \lambda \int_{0}^{t} s\left|u_{t}(s)\right|^{2} d s=\int_{0}^{t}\left(\left|u_{t}(s)\right|^{2}+A\left(|u(s)|_{1}^{2}\right)\right) d s, \\
t \geqq 0 .
\end{array}
$$

Note the following inequality

$$
a_{0}|u(t)|_{1}^{2} \leqq A\left(|u(t)|_{1}^{2}\right) \leqq m_{0}|u(t)|_{1}^{2}, \quad t \geqq 0,
$$

where $m_{0}=\sup _{0 \leqq r \leqq C^{2}} a(r)$. Hence, by virtue of (3.21) and (3.23), the right-hand side of (4.3) is bounded by a positive constant; which shows (4.2) for $j=0$.

In order to prove (4.2) for $j=1$, we introduce a pair of two nonnegative functions $\left\{\phi_{1}(t), \psi_{1}(t)\right\} ; \phi_{1}(t) \in C^{3}[0, \infty)$ is a monotone increasing function and $\psi_{1}(t) \in C^{3}[0, \infty)$ is an auxiliary function of $\phi_{1}(t)$.

Multiplying $(1.1)^{\prime}$ by $-\phi_{1}(t) \Delta \bar{u}_{t}$ and integrating over $R_{x}^{n} \times\left[t_{1}, t\right]$, we have 


$$
\begin{aligned}
\phi_{1}(t)\left(\left|u_{t}(t)\right|_{1}^{2}+a\left(|u(t)|_{1}^{2}\right)|u(t)|_{2}^{2}\right)+2 \lambda \int_{t_{1}}^{t} \phi_{1}(s)\left|u_{t}(s)\right|_{1}^{2} d s \\
=\phi_{1}\left(t_{1}\right)\left(\left|u_{t}\left(t_{1}\right)\right|_{1}^{2}+a\left(\left|u\left(t_{1}\right)\right|_{1}^{2}\right)\left|u\left(t_{1}\right)\right|_{2}^{2}\right) \\
\quad+2 \int_{t_{1}}^{t} \phi_{1}(s) a^{\prime}\left(|u(s)|_{1}^{2}\right) \operatorname{Re}\left(\operatorname{grad} u(s), \operatorname{grad} u_{t}(s)\right)|u(s)|_{2}^{2} d s \\
\quad+\int_{t_{1}}^{t} \phi_{1}^{\prime}(s)\left(\left|u_{t}(s)\right|_{1}^{2}+a\left(|u(s)|_{1}^{2}\right)|u(s)|_{2}^{2}\right) d s
\end{aligned}
$$

for $t \geqq t_{1} \geqq 0$. Next multiplying $(1.1)^{\prime}$ by $-\psi_{1}(t) \Delta \bar{u}$ and integrating over $R_{x}^{n} \times\left[t_{1}, t\right]$, we get

$$
\begin{aligned}
2 \psi_{1}(t) \operatorname{Re}\left(\operatorname{grad} u_{t}(t), \operatorname{grad} u(t)\right)+\left(\lambda \psi_{1}(t)-\psi_{1}^{\prime}(t)\right)|u(t)|_{1}^{2} \\
\quad+2 \int_{t_{1}}^{t} \psi_{1}(s) a\left(|u(s)|_{1}^{2}\right)|u(s)|_{2}^{2} d s+\int_{t_{1}}^{t}\left(\psi_{1}^{\prime \prime}(s)-\lambda \psi_{1}^{\prime}(s)\right)|u(s)|_{1}^{2} d s \\
=2 \psi_{1}\left(t_{1}\right) \operatorname{Re}\left(\operatorname{grad} u_{t}\left(t_{1}\right), \operatorname{grad} u\left(t_{1}\right)\right) \\
\quad+\left(\lambda \psi_{1}\left(t_{1}\right)-\psi_{1}^{\prime}\left(t_{1}\right)\right)\left|u\left(t_{1}\right)\right|_{1}^{2}+2 \int_{t_{1}}^{t} \psi_{1}(s)\left|u_{t}(s)\right|_{1}^{2} d s
\end{aligned}
$$

for $t \geqq t_{1} \geqq 0$.

Addition of (4.4) and (4.5) leads to the following identity:

$$
\begin{aligned}
\phi_{1}(t) a\left(|u(t)|_{1}^{2}\right)|u(t)|_{2}^{2}+\phi_{1}(t)\left|u_{t}(t)\right|_{1}^{2}+2 \psi_{1}(t) \operatorname{Re}\left(\operatorname{grad} u_{t}(t), \operatorname{grad} u(t)\right) \\
\quad+\left(\lambda \psi_{1}(t)-\psi_{1}^{\prime}(t)\right)|u(t)|_{1}^{2}+\int_{t_{1}}^{t}\left(\psi_{1}^{\prime \prime}(s)-\lambda \psi_{1}^{\prime}(s)\right)|u(s)|_{1}^{2} d s \\
\quad+\int_{t_{1}}^{t}\left(2 \lambda \phi_{1}(s)-\phi_{1}^{\prime}(s)-2 \psi_{1}(s)\right)\left|u_{t}(s)\right|_{1}^{2} d s \\
\quad+\int_{t_{1}}^{t}\left\{\left(2 \psi_{1}(s)-\phi_{1}^{\prime}(s)\right) a\left(|u(s)|_{1}^{2}\right)\right. \\
\left.\quad-2 \phi_{1}(s) a^{\prime}\left(|u(s)|_{1}^{2}\right) \operatorname{Re}\left(\operatorname{grad} u(s), \operatorname{grad} u_{t}(s)\right)\right\}|u(s)|_{2}^{2} d s \\
=\phi_{1}\left(t_{1}\right) a\left(\left|u\left(t_{1}\right)\right|_{1}^{2}\right)\left|u\left(t_{1}\right)\right|_{2}^{2}+\phi_{1}\left(t_{1}\right)\left|u_{t}\left(t_{1}\right)\right|_{1}^{2} \\
\quad+2 \psi_{1}\left(t_{1}\right) \operatorname{Re}\left(\operatorname{grad} u_{t}\left(t_{1}\right), \operatorname{grad} u\left(t_{1}\right)\right)+\left(\lambda \psi_{1}\left(t_{1}\right)-\psi_{1}^{\prime}\left(t_{1}\right)\right)\left|u\left(t_{1}\right)\right|_{1}^{2}
\end{aligned}
$$

for $t \geqq t_{1} \geqq 0$. Setting $\phi_{1}(t)=t$ and $\psi_{1}(t)=\lambda t / 2$ in (4.6) and making $t_{1}$ large enough $\left(\lambda t_{1}>1\right)$, we have, in view of (4.1) and (4.2) $(j=0)$,

$$
\begin{gathered}
a_{0} t|u(t)|_{2}^{2}+\frac{1}{2} t\left|u_{t}(t)\right|_{1}^{2}+\frac{1}{2} t\left(\left|u_{t}(t)\right|_{1}-\lambda|u(t)|_{1}\right)^{2}+\int_{t_{1}}^{t}(\lambda s-1)\left|u_{t}(s)\right|_{1}^{2} d s \\
+\int_{t_{1}}^{t}\left\{(\lambda s-1) a_{0}-2 m_{1} C M_{0} s^{1 / 2}\right\}|u(s)|_{2}^{2} d s \leqq N_{1}, \quad t \geqq t_{1} \geqq 0,
\end{gathered}
$$

with some $N_{1}$, where $m_{1}=\sup _{0 \leqq r \leqq C^{2}}\left|a^{\prime}(r)\right|$. Consequently, (4.7), in particular, implies 


$$
\left|u_{t}(t)\right|_{1}^{2} \leqq N_{2}^{2} t^{-1}, \quad t>0
$$

with some $N_{2}>0$.

We shall return to the identity (4.6) in order to derive a better estimate than (4.7) by making use of (4.8). Reset $\phi_{1}(t)=t^{2}$ and $\psi_{1}(t)=\alpha_{1} t$ in (4.6), where $\alpha_{1}>1$ is a parameter. Then, (4.1), (4.2) $(j=0)$ and (4.8) assure the existence of an $N_{3}$ satisfying

$$
\begin{aligned}
& t^{2}\left(a_{0}|u(t)|_{2}^{2}+\left|u_{t}(t)\right|_{1}^{2}\right)-2 \alpha_{1} t\left|u_{t}(t)\right|_{1} \cdot|u(t)|_{1}+\alpha_{1} \lambda t|u(t)|_{1}^{2} \\
& \quad+2 \int_{t_{1}}^{t}\left\{\lambda s-\left(\alpha_{1}+1\right)\right\} s\left|u_{t}(s)\right|_{1}^{2} d s \\
& \quad+2 \int_{t_{1}}^{t}\left\{\left(\alpha_{1}-1\right) a_{0}-m_{1} M_{0} N_{2}\right\} s|u(s)|_{2}^{2} d s \\
& \leqq N_{3} \quad \text { for } t \geqq t_{1},
\end{aligned}
$$

so that, by choosing $\alpha_{1}(>1)$ such that $\left(\alpha_{1}-1\right) a_{0}>m_{1} M_{0} N_{2}$ and taking a sufficiently large $t_{1}$, we may conclude that (4.2) holds for $j=1$.

In order to show (4.2) for $j \geqq 2$, we emply the following identity: for any monotone increasing function $\phi_{j} \in C^{3}[0, \infty)$ and $\alpha_{j}>1 / 2$,

$$
\begin{aligned}
\phi_{j}(t)\left(\left|u_{t}(t)\right|_{j}^{2}+a\left(|u(t)|_{1}^{2}\right)|u(t)|_{j+1}^{2}\right)+2 \alpha_{j} \phi_{j}^{\prime}(t) \operatorname{Re}\left((-\Delta)^{j / 2} u_{t}(t),(-\Delta)^{j / 2} u(t)\right) \\
\quad+\alpha_{j}\left(\lambda \phi_{j}^{\prime}(t)-\phi_{j}^{\prime \prime}(t)\right)|u(t)|_{j}^{2}+\alpha_{j} \int_{t_{j}}^{t}\left(\phi_{j}^{\prime \prime \prime}(s)-\lambda \phi_{j}^{\prime \prime}(s)\right)|u(s)|_{j}^{2} d s \\
\quad+\int_{t_{j}}^{t}\left\{2 \lambda \phi_{j}(s)-\left(2 \alpha_{j}+1\right) \phi_{j}^{\prime}(s)\right\}\left|u_{t}(s)\right|_{j}^{2} d s \\
\quad+\int_{t_{j}}^{t}\left\{\left(2 \alpha_{j}-1\right) \phi_{j}^{\prime}(s) a\left(|u(s)|_{1}^{2}\right)\right. \\
\left.\quad-2 \phi_{j}(s) a^{\prime}\left(|u(s)|_{1}^{2}\right) \operatorname{Re}\left(\operatorname{grad} u(s), \operatorname{grad} u_{t}(s)\right)\right\}|u(s)|_{j+1}^{2} d s \\
=\phi_{j}\left(t_{j}\right)\left(\left|u_{t}\left(t_{j}\right)\right|_{j}^{2}+a\left(\left|u\left(t_{j}\right)\right|_{1}^{2}\right)\left|u\left(t_{j}\right)\right|_{j+1}^{2}\right) \\
\quad+2 \alpha_{j} \phi_{j}^{\prime}\left(t_{j}\right) \operatorname{Re}\left((-\Delta)^{j / 2} u_{t}\left(t_{j}\right),(-\Delta)^{j / 2} u\left(t_{j}\right)\right) \\
\quad+\alpha_{j}\left(\lambda \phi_{j}^{\prime}\left(t_{j}\right)-\phi_{j}^{\prime \prime}\left(t_{j}\right)\right)\left|u\left(t_{j}\right)\right|_{j}^{2}, \quad \text { for } t \geqq t_{j} \geqq 0,
\end{aligned}
$$

which is obtained by multiplying $(1.1)^{\prime}$ by $(-\Delta)^{j}\left(\phi_{j} \bar{u}_{t}+\alpha_{j} \phi_{j}^{\prime} \bar{u}\right)$ and integrating over $R_{x}^{n} \times\left[t_{j}, t\right]$. Notice that

$$
\mid a^{\prime}\left(\left|\left(\left.u(t)\right|_{1} ^{2}\right) \operatorname{Re}\left(\operatorname{grad} u(t), \operatorname{grad} u_{t}(t)\right)\right| \leqq m_{1} M_{0} M_{1} t^{-3 / 2}\right.
$$

holds for $t>0$ (by (4.2) $(j=0,1)$ ). Hence, setting $\phi_{j}(t)=t^{j+1}$ in (4.9) and taking a sufficiently large $t_{j}$, we can inductively prove (4.2) for $j \geqq 2$. Thus (2.5) is verified.

To see (2.6), it suffices to apply (2.5) to (1.1)'. 
Now we shall prove (2.7) when $a \in C^{2}[0, \infty)$. Set

$$
b(t)=a\left(|u(t)|_{1}^{2}\right) .
$$

Then it follows from (4.1), (4.2) and (2.6) that

$$
\left|b^{\prime}(t)\right| \leqq K t^{-3 / 2} \text { and }\left|b^{\prime \prime}(t)\right| \leqq K t^{-3 / 2}, \quad t>0,
$$

with some $K>0$. Differentiation of $(1.1)^{\prime}$ with respect to $t$ leads to the equation

$$
u_{t t t}-b(t) \Delta u_{t}+\lambda u_{t t}-b^{\prime}(t) \Delta u_{t}=0, \quad x \in R^{n}, \quad t \geqq 0 .
$$

Multiply (4.11) by $(-\Delta)^{j}\left\{t^{j+2} \bar{u}_{t t}+\beta_{j}(j+2) t^{j+1} \bar{u}_{t}\right\}$ with $\beta_{j}>1 / 2$ and integrate the resulting expression over $R_{x}^{n} \times[0, t]$. Then as in the proof of (4.2), we can show (with the use of (4.10)) that there exist positive constants $L_{j}$ such that

$$
t^{j+2}\left(\left|u_{t t}(t)\right|_{j}^{2}+\left|u_{t}(t)\right|_{j+1}^{2}\right) \leqq L_{j}, \quad t \geqq 0, j=0,1,2, \cdots,
$$

which asserts (2.7). Thus the proof is complete.

Remark 4.1. Suppose that the data $\left(u_{0}, u_{1}\right)$ belong to $H^{k+2}\left(R_{x}^{n}\right) \times H^{k+1}\left(R_{x}^{n}\right)$ for $k \geqq 0$. As is easily seen from the proof of Theorem IV, it is possible to show (2.4), (2.5) for $0 \leqq j \leqq k+1$ and (2.6) for $0 \leqq j \leqq k$. Moreover, if $k \geqq 1$ and $a \in C^{2}[0, \infty)$, then (2.7) also holds true for $0 \leqq j \leqq k$.

4.2. Proof of Corollary 2.2.

Note the following well-known inequality due to Nirenberg [9];

$$
\|u\|_{\infty} \leqq c_{0}|u|_{m}^{\theta}\|u\|^{1-\theta} \quad \text { for } u \in H^{m}\left(R_{x}^{n}\right),
$$

where $m \geqq[n / 2]+1$ and $0<\theta=n / 2 m<1$. Then it follows from (2.4), (2.5) and (4.12) that

$$
\|u(t)\|_{\infty} \leqq c_{0}|u(t)|_{m}^{n / 2 m}\|u(t)\|^{1-(n / 2 m)} \leqq c_{0}^{\prime} t^{-n / 4},
$$

which implies (2.8). Other decay estimates (2.9) and (2.10) are derived from (2.5), (2.6) and (4.12) in the same way.

Remark 4.2. In Corollary 2.2, decay estimates (2.8)-(2.10) still remain true for the initial data $\left(u_{0}, u_{1}\right) \in H^{s+2}\left(R_{x}^{n}\right) \times H^{s+1}\left(R_{x}^{n}\right)$ with $s=[n / 2]+1$ (see Remark 4.1). 


\section{§. Some results on the mixed problem}

Let $\Omega$ be a bounded domain in $R_{x}^{n}$ with $C^{\infty}$ boundary $\Gamma$. We shall consider the following mixed problem

$$
\begin{array}{ll}
u_{t t}-a\left(\int_{\Omega}|\operatorname{grad} u(x, t)|^{2} d x\right) \Delta u+\lambda u_{t}=f, & x \in \Omega, \quad t \in[0, \infty), \\
u(x, t)=0, & x \in \Gamma, \quad t \in[0, \infty), \\
u(x, 0)=u_{0}(x), & x \in \Omega, \\
u_{t}(x, 0)=u_{1}(x), & x \in \Omega,
\end{array}
$$

where $a$ is a function satisfying (A.1) and $\lambda$ is a positive constant (see Dickey [1], [2] and Pohozaev [10]).

Let $H^{s}(\Omega)$ be the usual Sobolev space of order $s$; the space of functions $u$ such that $u$ and all its derivatives of order $\leqq s$ belong to $L^{2}(\Omega)$. The closure of $C_{0}^{\infty}(\Omega)$ in $H^{s}(\Omega)$ is written by $H_{0}^{s}(\Omega)$. As in the preceding sections, we denote by $\|\cdot\|_{s}$ (resp. $\left.\|\cdot\|\right) H^{s}(\Omega)$-norm (resp. $L^{2}(\Omega)$-norm).

We define a positive self-adjoint operator $A$ in $L^{2}(\Omega)$ by $A u=-\Delta u$ with domain $D(A)=H^{2}(\Omega) \cap H_{0}^{1}(\Omega)$. It is well known that $D\left(A^{1 / 2}\right)=H_{0}^{1}(\Omega)$ and $\left\|A^{1 / 2} u\right\|=\|\operatorname{grad} u\|\left(u \in D\left(A^{1 / 2}\right)\right.$. So the mixed problem (5.1)-(5.4) can be written in an abstract form

$$
\left\{\begin{array}{l}
u_{t}(t)+a\left(\left\|A^{1 / 2} u(t)\right\|^{2}\right) A u(t)+\lambda u_{t}(t)=f(t), \quad t \geqq 0, \\
u(0)=u_{0}, \\
u_{t}(0)=u_{1} .
\end{array}\right.
$$

Repeating the arguments in $\S 3$ with a slight modification, we can obtain the similar existence results on the mixed problem (5.1)-(5.4). We shall state them without proofs.

Theorem 5.1. Let $u_{0} \in H^{2}(\Omega) \cap H_{0}^{1}(\Omega), u_{1} \in H_{0}^{1}(\Omega)$ and $f \in C\left([0, \infty) ; H_{0}^{1}(\Omega)\right)$. Then there exists a positive constant $T_{0}$ such that the mixed problem (5.1)(5.4) has a unique solution $u$ on $\left[0, T_{0}\right]$ satisfying

$$
u \in C\left(\left[0, T_{0}\right] ; H^{2}(\Omega) \cap H_{0}^{1}(\Omega)\right) \cap C^{1}\left(\left[0, T_{0}\right] ; H_{0}^{1}(\Omega)\right) \cap C^{2}\left(\left[0, T_{0}\right] ; L^{2}(\Omega)\right) .
$$

Theorem 5.2. There exists a positive number $\delta_{0}$ (which depends on a, $a^{\prime}$ and $\left.\lambda\right)$ such that, if the data $\left(u_{0}, u_{1}, f\right) \in H^{2}(\Omega) \cap H_{0}^{1}(\Omega) \times H_{0}^{1}(\Omega) \times C([0, \infty)$; $\left.H_{0}^{1}(\Omega)\right)$ satisfy

$$
\left\|u_{0}\right\|_{2} \leqq \delta_{0}, \quad\left\|u_{1}\right\|_{1} \leqq \delta_{0} \quad \text { and } \quad \int_{0}^{\infty}\|f(s)\|_{1} d s \leqq \delta_{0},
$$


then the mixed problem (5.1)-(5.4) has a unique solution $u$ on $[0, \infty)$ such that $u \in C\left([0, \infty) ; H^{2}(\Omega) \cap H_{0}^{1}(\Omega)\right) \cap C^{1}\left([0, \infty) ; H_{0}^{1}(\Omega)\right) \cap C^{2}\left([0, \infty) ; L^{2}(\Omega)\right)$.

Furthermore,

$$
\sup _{t \geqq 0}\|u(t)\|_{2}<\infty \quad \text { and } \sup _{t \geqq 0}\left\|u_{t}(t)\right\|_{1}<\infty .
$$

TheOREm 5.3. Let a belong to the class $C^{k+1}[0, \infty)(k \geqq 1)$ and let $\delta_{0}$ be the positive number in Theorem 5.2. If the data $\left(u_{0}, u_{1}, f\right)$ satisfy

$$
\begin{array}{cc}
u_{0} \in D\left(A^{(k-2) / 2}\right), \quad u_{1} \in D\left(A^{(k+1) / 2}\right), \quad A^{(k+1-i) / 2} f \in C^{i}\left([0, \infty) ; L^{2}(\Omega)\right), \\
(i=0,1,2, \cdots, k)
\end{array}
$$

and (5.5), then the mixed problem (5.1)-(5.4) has a unique solution $u$ on $[0, \infty)$ satisfying

$$
A^{(k+2-i) / 2} u \in C^{i}\left([0, \infty) ; L^{2}(\Omega)\right), \quad i=0,1,2, \cdots, k+2 .
$$

Remark 5.1. From $A^{(k+2-i) / 2} u \in C^{i}\left([0, \infty) ; L^{2}(\Omega)\right)$ for $0 \leqq i \leqq k+1$, it is easily seen that $u$ belongs to the class $C^{i}\left([0, \infty) ; H^{k+2-i}(\Omega) \cap H_{0}^{1}(\Omega)\right.$ ) for $0 \leqq i \leqq k+1$.

Remark 5.2. Pohozaev [10] has approached the mixed problem (5.1)(5.4) with $\lambda=0$ via the Galerkin's method. He has shown that there exists a global solution $u$ of (5.1)-(5.4) if the data $\left(u_{0}, u_{1}, f\right)$ are contained in some special classes of functions. See also Lions [4].

Finally we shall study the asymptotic behavior of global solutions. For simplicity, we assume that $a \in C^{\infty}[0, \infty), f \equiv 0$ and that the initial data $\left(u_{0}, u_{1}\right) \in C_{0}^{\infty}(\Omega) \times C_{0}^{\infty}(\Omega)$ satisfy (5.5). (Note that both $u_{0}$ and $u_{1}$ are in $D\left(A^{k / 2}\right)$ for any $k \geqq 0$.) Then making use of the weighted energy method developed in $\S 4$, we can obtain the following exponential decay of solutions. (The key point of deriving the exponential decay lies in the use of Poincaré's inequality

$$
\left\|A^{1 / 2} u\right\| \geqq c_{0}\|u\| \quad \text { for } u \in D\left(A^{1 / 2}\right)=H_{0}^{1}(\Omega)
$$

with some $c_{0}>0$.) See also Yamada [12].

TheOREm 5.4. Let $u$ be a solution of (5.1)-(5.4) with $f \equiv 0$. Then there exists a positive constant $\alpha>0$ such that

$$
\left\|A^{(i+1) / 2} u(t)\right\|^{2}+\left\|A^{j / 2} u_{t}(t)\right\|^{2}=O\left(e^{-\alpha t}\right) \text { as } t \rightarrow \infty,
$$

for every $j \geqq 0$. 


\section{REFERENCES}

[1] R. W. Dickey, Infinite systems of nonlinear oscillation equations related to the string, Proc. Amer. Math. Soc., 23 (1969), 459-468.

[2] - Infinite systems of nonlinear oscillation equations with linear damping, SIAM J. Appl. Math., 19 (1970), 208-214.

[3] _ The initial value problem for a nonlinear semi-infinite string, Proc. Roy. Soc. Edinburgh, 82 (1978), 19-26.

[ 4 ] J. L. Lions, On some questions in boundary value problems of mathematical physics, Contemporary Developments in Continuum Mechanics and Partial Differential Equations, (ed. by G. M. de La Penha and L. A. Medeiros), North-Holland, 1978.

[5] A. Matsumura, Global existence and asymptotics of the solutions of the secondorder quasilinear hyperbolic equations with the first order dissipation, Publ. RIMS, Kyoto Univ., 13 (1977), 349-379.

[6] G. P. Menzala, Une solution d'une équation non linéaire d'évolution, C. R. Acad. Sc. Paris, 286 (1978), 273-275.

[7] - On classical solutions of a quasilinear hyperbolic equation, Memórias de Matemática da Universidade Federal do Rio de Janeiro, 1978.

[ 8 ] S. Mizohata, The Theory of Partial Differential Equations, Cambridge University Press, 1973.

[9] L. Nirenberg, On elliptic partial differential equations, Ann. Scuola. Norm. Sup. Pisa, 13 (1959), 115-162.

[10] S. I. Pohozaev, On a class of quasilinear hyperbolic equations, Math. USSR Sbornik, 25 (1975), 145-158.

[11] S. L. Sobolev, Applications of Functional Analysis in Mathematical physics, Transl. Math. Monographs Vol. 7, A.M.S., 1963.

[12] Y. Yamada, On the decay of solutions for some nonlinear evolution equations of second order, Nagoya Math. J., 73 (1979), 69-98.

Department of Mathematics

Faculty of Science

Nagoya University

Chikusa-ku, Nagoya, 464, Japan 